\title{
Combination of Salivary Gland Ultrasonography and Virtual Touch Quantification for Diagnosis of Sjögren's Syndrome: A Preliminary Study
}

\author{
Shaoqi Chen,' ${ }^{1}$ Yukai Wang, ${ }^{2}$ Guohong Zhang, ${ }^{3}$ and Shigao Chen ${ }^{4}$ \\ ${ }^{1}$ Department of Ultrasound, The First Affiliated Hospital of Shantou University Medical College, Shantou, Guangdong, China \\ ${ }^{2}$ Department of Rheumatology, Shantou Central Hospital, Shantou, Guangdong, China \\ ${ }^{3}$ Department of Pathology, Shantou University Medical College, Shantou, Guangdong, China \\ ${ }^{4}$ Department of Radiology, Mayo Clinic College of Medicine, Rochester, MN, USA
}

Correspondence should be addressed to Yukai Wang; stzxyywyk@126.com and Guohong Zhang; g_ghzhang@stu.edu.cn

Received 21 July 2016; Accepted 23 November 2016

Academic Editor: Sadik A. Khuder

Copyright (C) 2016 Shaoqi Chen et al. This is an open access article distributed under the Creative Commons Attribution License, which permits unrestricted use, distribution, and reproduction in any medium, provided the original work is properly cited.

\begin{abstract}
A total of 136 subjects (51 SS patients, 35 sicca syndrome patients without SS, and 50 healthy volunteers) were enrolled in this study. The mean SWV value for salivary glands of SS patients was statistically higher than that of controls $(2.81 \pm 0.66 \mathrm{~m} / \mathrm{s}$ versus $1.85 \pm$ $0.28 \mathrm{~m} / \mathrm{s}$ for parotid glands and $2.29 \pm 0.34 \mathrm{~m} / \mathrm{s}$ versus $1.82 \pm 0.25 \mathrm{~m} / \mathrm{s}$ for submandibular glands, resp.). Combining SWV values of parotid and submandibular glands gives a sensitivity of $88.2 \%$ (95\% CI: 76.1-95.6\%) and specificity of 96.0\% (95\% CI: 86.3-99.5\%) at the cutoff point of $2.19 \mathrm{~m} / \mathrm{s}$, with an AUROC of 0.954 (95\% CI: 0.893-0.986). In addition, combining SGUS score and SWV value yields a sensitivity of $98.0 \%$ (95\% CI: 89.6-100\%), specificity of 90.0\% (95\% CI: 78.2-96.7\%), and AUROC of 0.962 (95\% CI: 0.9040.990). Classification tree considering the sequential use of SGUS score and SWV value achieved $92.1 \%$ accuracy for diagnosis of SS. Similarly, the ROC curve of combined SGUS scores and SWV values yields an AUROC of 0.954 (95\% CI: 0.885-0.987), sensitivity of $97.1 \%$ (95\% CI: 85.1-99.9\%), and specificity of 92.2\% (95\% CI: 81.1-97.8\%) for separating sicca syndrome patients (without SS) from SS patients. Combining SGUS and VTQ provides a promising tool for diagnosis of SS.
\end{abstract}

\section{Introduction}

Sjögren's syndrome (SS) is a common chronic autoimmune disease which affects mainly the exocrine glands with a typical focal lymphocytic infiltration, potentially leading to xerostomia and xerophthalmia [1]. SS is commonly found in patients with rheumatic disorders affecting the head and neck [2]. Currently, SS is diagnosed according to the criteria proposed by the American-European Consensus Group (AECG) in 2002 and American College of Rheumatology (ACR) in 2012 [3, 4]. An international consensus classification criterion for SS, approved by AECG and ACR, was recently published [5]. Labial salivary gland biopsy plays an important role in the diagnosis of SS. However, salivary gland biopsy is an invasive procedure associated with patient discomfort and the risk of complications, and disparity in minor salivary gland biopsy evaluation can lead to overestimation of SS [6]. In recent years, salivary gland ultrasonography (SGUS) has been proposed as a convenient and noninvasive alternative to sialography, sialoscintigraphy, and magnetic resonance imaging for diagnosis and classification of primary and secondary SS [7-14]. Several comprehensive SGUS scoring systems for diagnosis of SS have been developed previously [15]. More recently, a simplified scoring system based on parenchyma ultrasound heterogeneity was proposed, which is relatively easy to use $[16,17]$. However, these scoring systems are semiquantitative at best and somewhat subjective, which may lead to operator-dependent results. Therefore, there is still a need for noninvasive and objective methods for diagnosing SS salivary glands.

Shear wave velocity (SWV) imaging is an emerging ultrasound imaging modality which quantitatively evaluates tissue stiffness, a biomarker closely related to pathology. Typically, shear waves are generated in tissue by ultrasound 
radiation force and the shear wave propagation velocity is measured and used alone or converted to Young's modulus for quantitative evaluation of tissue stiffness [18]. This imaging modality has shown good promise for liver fibrosis staging and cancer diagnosis [19]. A recent study indicates that SWV of parotid gland is a potentially useful parameter for diagnosis of primary SS [20]. However, combining SGUS score and salivary gland SWV value for SS classification has not been reported.

Because B-mode image texture and tissue stiffness represent different aspects of tissue characteristics, it is expected that the combination of both texture and stiffness information may improve the diagnostic performance. The aim of the present study is to investigate the value of combining simplified SGUS score and SWV value obtained with Virtual Touch Quantification (VTQ) in salivary glands for SS diagnosis.

\section{Methods}

2.1. Patients and Healthy Controls. This study was approved by the Human Research Ethics Committee of Shantou Central Hospital and Shantou University Medical College. Written, informed consent was obtained for all participants. Between January 2014 and February 2015, 51 consecutive SS patients (including primary and secondary SS) were enrolled in this study. Diagnosis of SS was made according to the AECG criteria, which includes a standardized clinical examination performed by an experienced rheumatologist (Yukai Wang), serological and laboratory tests, ocular tests, and salivary gland biopsy. During the same period of time, 50 healthy adult volunteers without SS were recruited as the control group and examined by SGUS and VTQ. All healthy volunteers had a normal medical history and physical examination; had no symptoms of xerostomia or keratoconjunctivitis sicca; had no sialoadenitis or mass lesions in salivary glands; and were not using medications. In addition, 35 sicca syndrome patients (without SS) were studied and compared with the SS patients.

2.2. SGUS Score. An Acuson S2000 ultrasound system (Siemens Medical Solutions, Mountain View, CA, USA) equipped with a linear 4-9 $\mathrm{MH}$ probe was used in this study. When scanned, subjects were in a supine position and the neck extended and the head turned to the opposite side. The ultrasound transducer was gently coupled to the body surface with a sufficient amount of ultrasound gel. The bilateral parotid glands and submandibular glands were imaged. Parenchymal homogeneity was graded from 0 to 3 in accordance with the US scoring system described by Theander and Mandle and Hočvar et al. [16, 21]: score $0=$ complete homogeneity, score 1 $=$ mild inhomogeneity, score $2=$ evident inhomogeneity, and score 3 = gross inhomogeneity. The final score was selected as the highest score among the 4 salivary glands [16]. All ultrasound acquisitions and scoring were performed by the same physician (Shaoqi Chen).

2.3. Virtual Touch Quantification (VTQ). Measurement of shear wave velocity was also obtained with the S2000 using the VTQ function. The bilateral parotid glands and submandibular glands were identified under the guidance of real-time B-mode imaging. Six VTQ measurements were obtained in the central, peripheral, and subcapsular areas (2 measurements at each area) without visible vessels of each salivary gland during breathhold. Each VTQ measurement gave an estimate of shear wave velocity (SWV) in $\mathrm{m} / \mathrm{s}$. In order to reduce measurement variation, the highest and lowest SWV of all 6 measurements were eliminated and the remaining 4 measurements were averaged and used as the final value for each salivary gland. All VTQ measurements were performed by the same physician (Shaoqi Chen).

2.4. Statistical Analysis. Continuous variables were expressed as mean \pm standard deviation (SD). Comparison between SS and controls was performed by Fisher's exact chi-square test. The data were statistically analyzed using the SPSS software version 16 (IBM SPSS Statistics, Armonk, NY, USA). The optimal cutoff values were determined from a receiver operating characteristic curves (ROC) using MedCalc statistical software version 12.3.0. The sensitivity, specificity, and area under the ROC curve (AUROC) were used as diagnostic performance indicators. The optimal cutoff point was identified according to Youden tests. A classification and regression tree (CART) was used to determine whether separation between controls and SS patients could be improved by combining SGUS score and SWV value. Leave-one-out cross-validation (10-fold) was applied to determine classifier performance.

\section{Results}

The SS group included 51 patients ( 1 male and 50 female) with mean age of $47.0 \pm 12.6$ years (range: $23-77$ years). The control group included 50 healthy individuals ( 2 male and 48 female) with a mean age of $45.3 \pm 12.2$ years (range: $20-71$ years). There were 35 ( 5 male and 30 female) sicca syndrome patients (without SS) with a mean age of $52.5 \pm 12.5$ years (range: 24-73 years). There was no statistically significant difference between the patient and control groups in terms of age and gender $(P>0.05)$.

3.1. Difference of SGUS Score between SS Patients and Healthy Controls. Representative B-mode images with SGUS score of 0 through 3 are shown in Figure 1. The SS patients presented SGUS scores covering all 4 categories: $7.8 \%$ for score $0,35.3 \%$ for score $1,47.1 \%$ for score 2 , and $9.8 \%$ for score 3 . In contrast, score 0 accounted for $92 \%$ of the controls, and score 1 was detected in $8 \%$ of controls. The difference in SGUS scores between SS patients and controls was highly significant $(P<$ 0.001).

\subsection{Diagnostic Performance of SWV Value for Separating SS} Patients and Healthy Controls. Representative images of the parotid gland with VTQ measurements are shown in Figure 2. The mean SWV value for parotid glands of SS patients was statistically higher than that of controls $(2.81 \pm 0.66 \mathrm{~m} / \mathrm{s}$ versus $1.85 \pm 0.28 \mathrm{~m} / \mathrm{s}, P<0.0001)$. The ROC curve of SWV for separating parotid glands of SS patients from those 

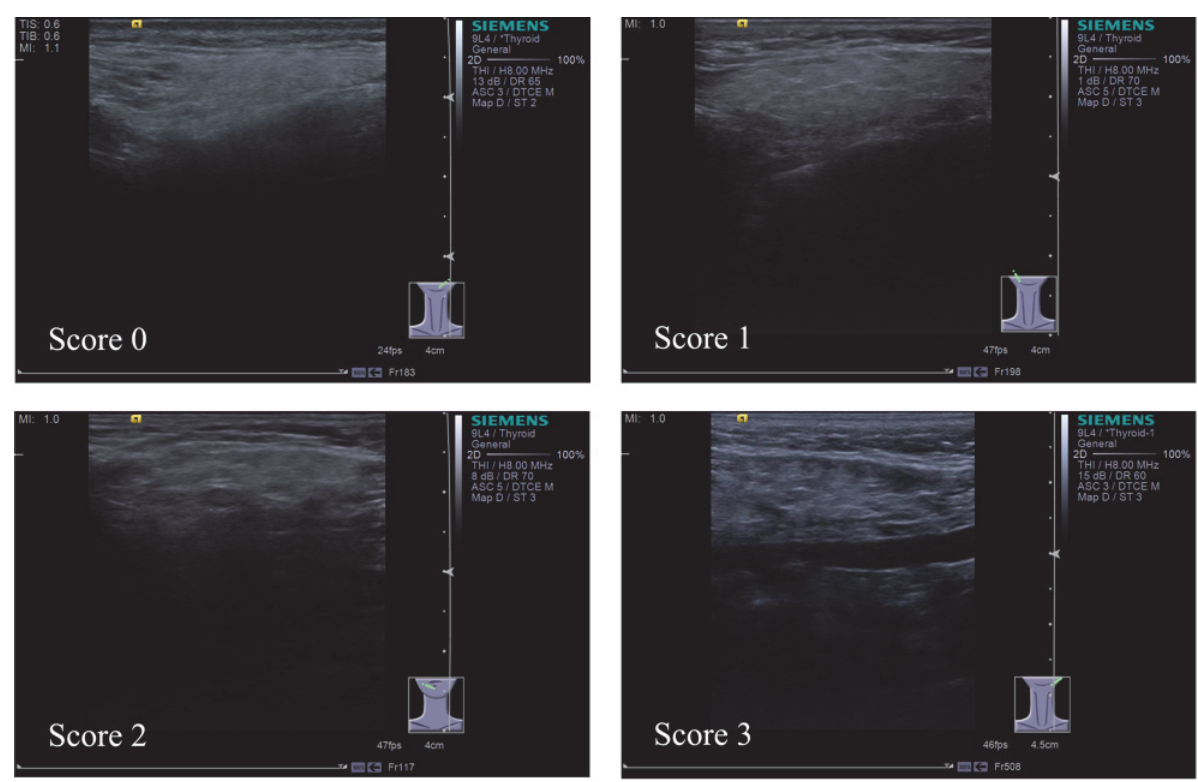

FIGURE 1: Representative B-mode ultrasound images for salivary glands: score $0=$ complete homogeneity, score $1=$ mild inhomogeneity, score $2=$ evident inhomogeneity, and score 3 = gross inhomogeneity.

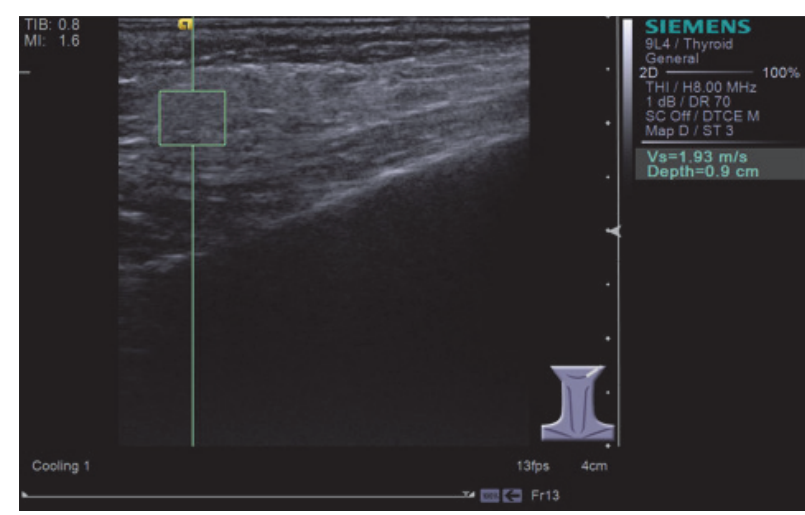

(a)

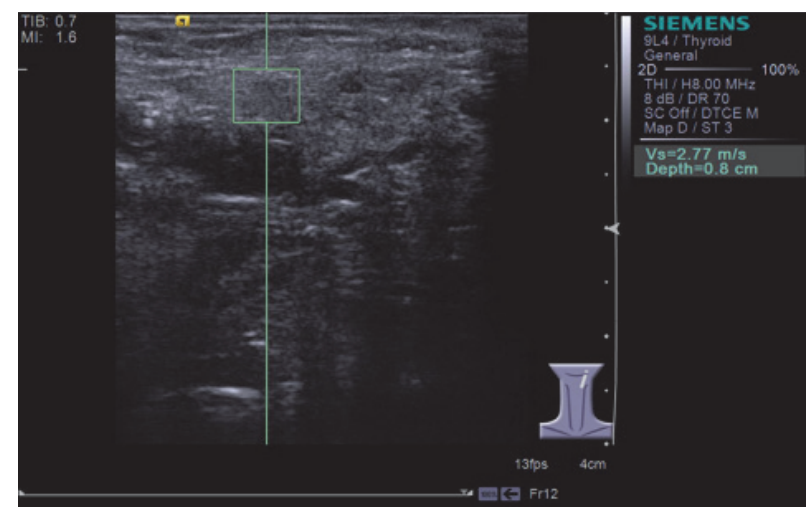

(b)

FIGURE 2: Representative images of parotid glands with shear wave velocity (SWV) measurements using Virtual Touch Quantification (VTQ). (a) SWV value was $1.93 \mathrm{~m} / \mathrm{s}$ in a healthy volunteer and (b) $2.77 \mathrm{~m} / \mathrm{s}$ in a SS patient.

of healthy controls is shown in Figure 3(a). The AUROC was 0.945 , with a $95 \%$ confidence interval (CI) of 0.882 0.981 . Using a cutoff value of $2.07 \mathrm{~m} / \mathrm{s}$, the sensitivity and specificity were $92.2 \%$ (95\% CI: 81.1-97.8\%) and $88.0 \%$ (95\% CI: 75.7-95.5\%), respectively. Similarly, the mean SWV value for submandibular glands of SS patients was significantly higher than that of controls $(2.29 \pm 0.34 \mathrm{~m} / \mathrm{s}$ versus $1.82 \pm$ $0.25 \mathrm{~m} / \mathrm{s}, P<0.0001)$. The ROC curve of SWV for separating submandibular glands of SS patients from those of healthy controls is shown in Figure 3(b). The AUROC was 0.854 (95\% CI: $0.770-0.916$ ). With a cutoff value of $2.06 \mathrm{~m} / \mathrm{s}$, the sensitivity and specificity were $76.5 \%$ (95\% CI: $62.5-87.2 \%$ ) and $92.0 \%$ (95\% CI: 80.8-97.8\%), respectively. In SS patients, the mean SWV value of parotid gland was significantly higher than that of the submandibular gland $(2.81 \pm 0.66 \mathrm{~m} / \mathrm{s}$ versus $2.29 \pm 0.34 \mathrm{~m} / \mathrm{s}, P<0.001)$.
After combining results of parotid and submandibular glands (by averaging), the mean SWV value of SS patients $(2.55 \pm 0.41 \mathrm{~m} / \mathrm{s})$ differed significantly from that of controls $(1.83 \pm 0.20 \mathrm{~m} / \mathrm{s})(P<0.001)$. The ROC curve for separating SS patients from healthy controls is shown in Figure 3(c). AUROC was 0.954 (95\% CI: 0.893-0.986). With a cutoff value of $2.19 \mathrm{~m} / \mathrm{s}$, the sensitivity and specificity were $88.2 \%$ (95\% CI: 76.1-95.6\%) and 96.0\% (95\% CI: 86.3-99.5\%), respectively.

3.3. Diagnostic Performance of Combining SGUS Score and SWV Value for Separating SS Patients and Healthy Controls. A classification tree analysis based on SGUS scores and SWV values was used for distinguishing SS patients from healthy controls. The classification tree included two classification nodes and three terminal nodes (Figure 4). The first node was separated by SGUS score, and the second node was split by 


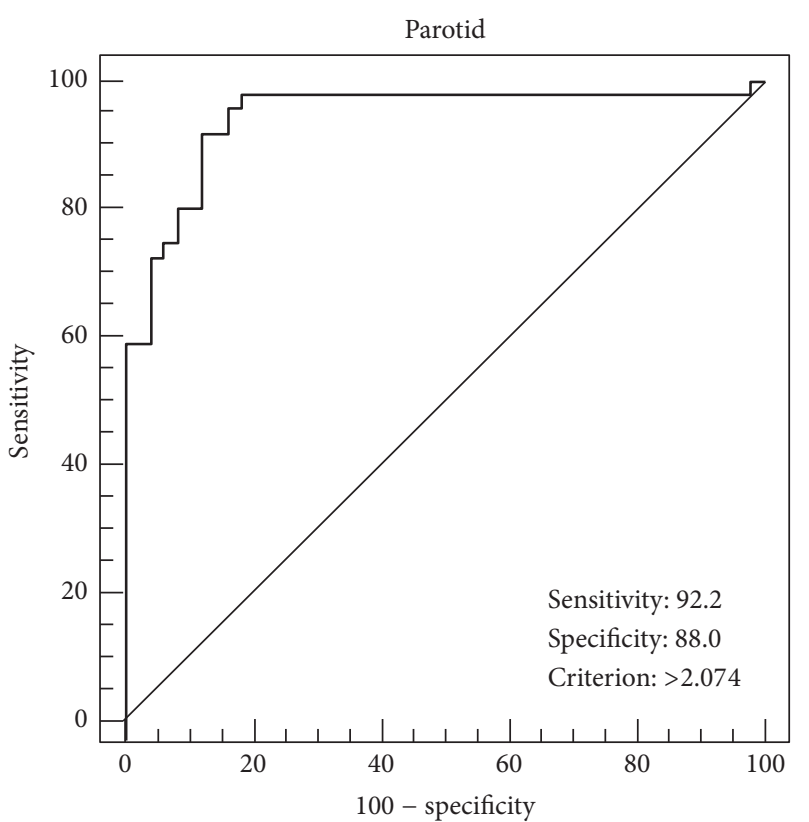

(a)

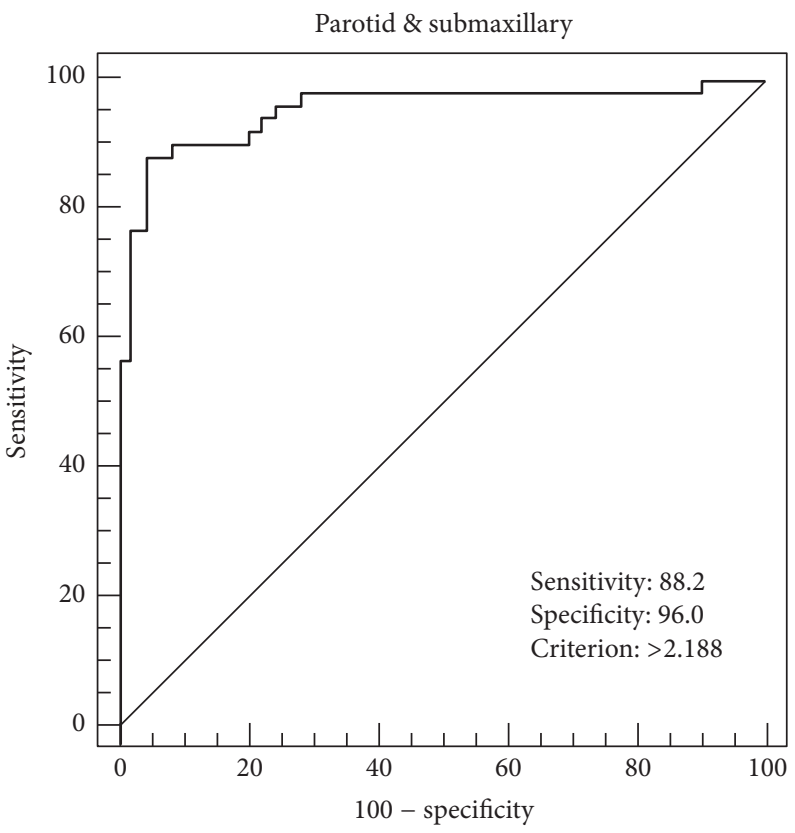

(c)

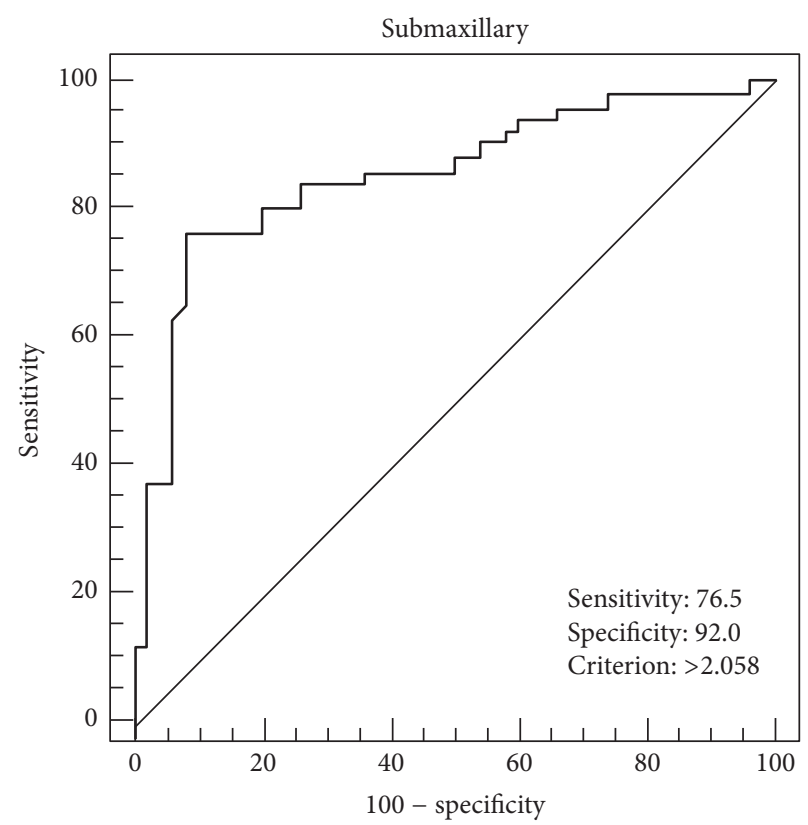

(b)

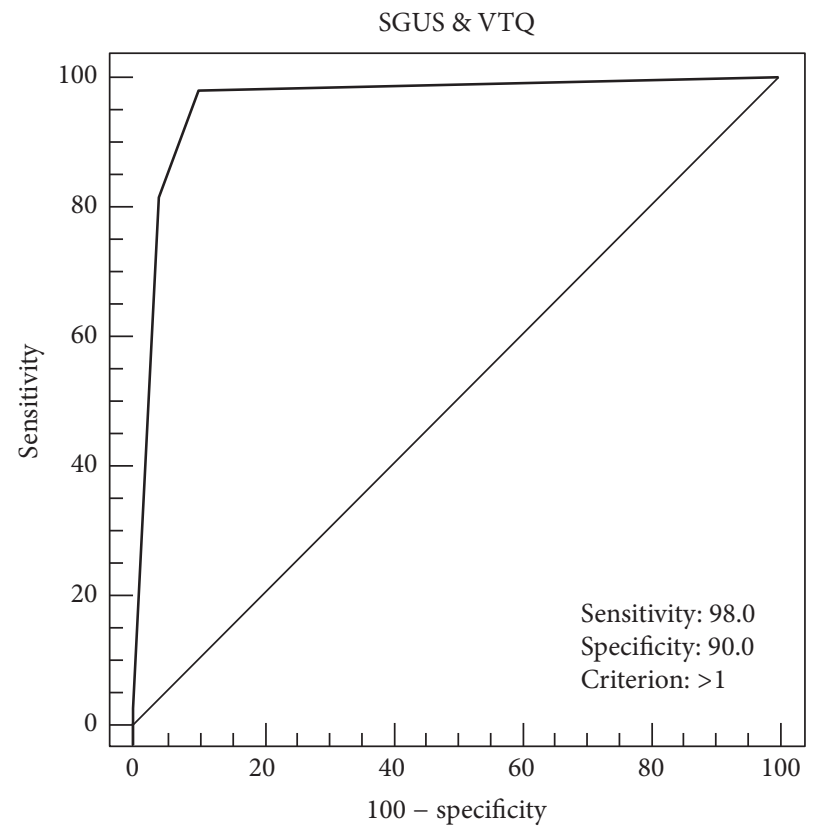

(d)

FIGURE 3: ROC curves with the optimal cutoff value, sensitivity, and specificity using (a) shear wave velocity (SWV) value of parotid glands; (b) SWV value of submandibular glands; (c) mean SWV value of parotid and submandibular glands; and (d) combination of SGUS score and mean SWV value.

VTQ value, with a threshold of $2.19 \mathrm{~m} / \mathrm{s}$. The mean accuracy and error of the classification tree estimated by performing 10 -fold cross-validation were $92.1 \%$ and 0.03 .

The ROC curve for separating SS patients from healthy controls using the classification tree is shown in Figure 3(d). AUROC was 0.962 (95\% CI: 0.904-0.990). With a SWV cutoff value of $2.19 \mathrm{~m} / \mathrm{s}$, the sensitivity and specificity were 98.0\% (95\% CI: 89.6-100\%) and 90.0\% (95\% CI: 78.2-96.7\%), respectively.
3.4. Diagnostic Performance of Separating SS Patients and Sicca Syndrome Patients. The mean SWV values for parotid and submandibular glands of sicca syndrome patients (without SS) were $1.93 \pm 0.28 \mathrm{~m} / \mathrm{s}$ and $1.84 \pm 0.17 \mathrm{~m} / \mathrm{s}$, respectively. After combining values of parotid and submandibular glands, the mean SWV value of sicca syndrome patients $(1.88 \pm$ $0.15 \mathrm{~m} / \mathrm{s}$ ) differed significantly from that of patients with SS $(P<0.001)$. The ROC curve for separating sicca syndrome patients from SS patients had a sensitivity of $97.1 \%$ (95\% CI: 


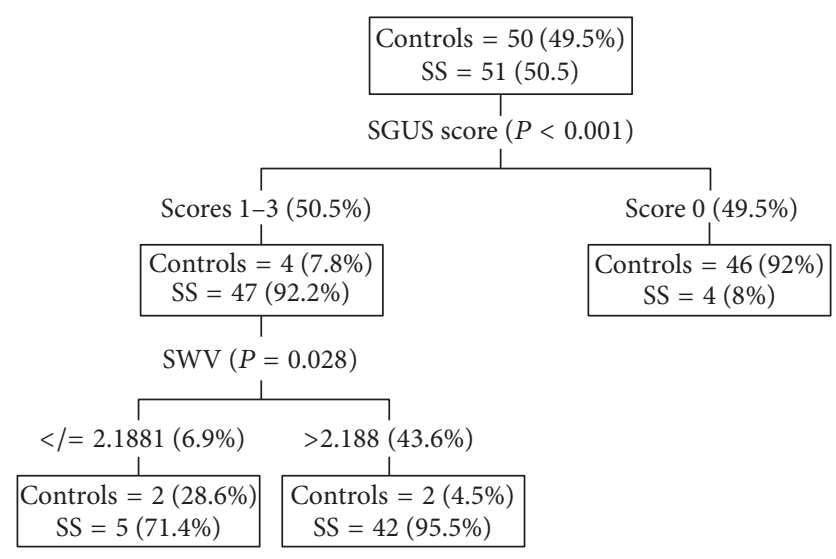

FIGURE 4: Classification tree for combination of SGUS score and mean SWV value. Each terminal node specifies numbers and percentages of SS and controls. The resulting correct classification rate was $92.1 \%$.

85.1-99.9\%), specificity of $88.2 \%$ (95\% CI: 76.1-95.6\%), and AUROC of 0.952 (95\% CI: 0.884-0.987) with a cutoff value of $2.18 \mathrm{~m} / \mathrm{s}$.

Combining the SGUS scores and SWV values, the ROC curve yielded an AUROC of 0.954 (95\% CI: 0.885-0.987), sensitivity of $97.1 \%$ (95\% CI: $85.1-99.9 \%$ ), and specificity of 92.2\% (95\% CI: 81.1-97.8\%). The mean accuracy and error of the classification tree were $91.9 \%$ and 0.03 , respectively.

\section{Discussion}

The evaluation of salivary gland involvement contributes significantly to the diagnosis of SS. Therefore, development of noninvasive and accurate diagnostic strategies for salivary glands would significantly benefit SS patients. Our preliminary study demonstrates that VTQ may provide important information for diagnosis of SS.

In the study of Knopf et al. [20], the mean SWV values for the parotid and submandibular glands were $2.86 \mathrm{~m} / \mathrm{s}$ and $2.17 \mathrm{~m} / \mathrm{s}$, respectively, in primary SS patients, and $1.87 \mathrm{~m} / \mathrm{s}$ and $1.81 \mathrm{~m} / \mathrm{s}$, respectively, in healthy subjects. These values are very similar to results of our current study: $2.81 \mathrm{~m} / \mathrm{s}$ and $2.29 \mathrm{~m} / \mathrm{s}$ for parotid and submandibular glands, respectively, in SS patients, and $1.85 \mathrm{~m} / \mathrm{s}$ and $1.82 \mathrm{~m} / \mathrm{s}$, respectively, for healthy volunteers. However, the optimal cutoff value for parotid glands in our study $(2.074 \mathrm{~m} / \mathrm{s})$ was lower than that in the study of Knopf et al. $(2.395 \mathrm{~m} / \mathrm{s})$ [20]. This difference may be due to selection of different controls in these two studies: controls in the Knopf study were non-SS patients with sicca symptoms and/or salivary gland swelling, whereas controls in our study were healthy volunteers without any sicca or salivary gland symptoms.

Shear wave velocity measurements provide an objective and quantitative evaluation of tissue stiffness, which may add important information complementary to B-mode ultrasound homogeneity for diagnosis of SS. The combination of B-mode ultrasonography and SWV has been investigated in a study of radiation submaxillitis [22]. However, there are no prior reports on combination of SGUS score and SWV value in SS diagnosis. To the best of our knowledge, our study is the first research that investigates the diagnostic accuracy of combining simplified SGUS score and SWV value in classification of SS. Using a classification tree to combine SGUS score and SWV value, a mean accuracy of $92.1 \%$ could be achieved for separating SS patients from healthy volunteers in our study. A similar accuracy of 91.9 was achieved for separating sicca syndrome patients (without SS) from SS patients. SWV measurements are relatively easy to obtain during routine ultrasound imaging of the salivary glands. Cornec et al. [23] proposed that, in the diagnosis of primary SS, salivary gland biopsy should be performed only when the results of ultrasound imaging are negative. Therefore, the integration of SWV and SGUS score for improving the accuracy of ultrasound diagnosis may reduce the need of salivary gland biopsy in a substantial number of patients.

This study has several limitations. First, this is a singlecenter study with a relatively limited sample size, in which primary and secondary SS were not analyzed separately. Further studies with larger sample size are needed to confirm findings of this study. A large sample size would also allow the study of any potential correlations between SWV/SGUS and severity of SS. Second, sicca syndrome patients (without SS) had many different coexisting conditions, such as diabetes and chronic hepatitis. Future studies including nonSS patients without coexisting conditions may provide more insights into the diagnostic performance in a typical clinical setting.

In conclusion, the combination of SGUS and SWV value provides a promising tool in diagnosis of SS.

\section{Competing Interests}

The authors have no conflict of interests to disclose for this study.

\section{Acknowledgments}

This study was supported by the Guangdong Province Medical Science and Research Fund (Grant no. A201566); Foundation of the Development of Science and Technology of Guangdong Province (Grant no. 2017); Foundation for Young Talents in Higher Education of Guangdong, China (Grant no. 2015KQNCX048); and the Natural Science Foundation of Guangdong Province (Grant no. 2014A030307003).

\section{References}

[1] C. Baldini, R. Talarico, A. G. Tzioufas, and S. Bombardieri, "Classification criteria for Sjogren's syndrome: a critical review," Journal of Autoimmunity, vol. 39, no. 1-2, pp. 9-14, 2012.

[2] A. Knopf, M. Bas, A. Chaker et al., "Rheumatic disorders affecting the head and neck: underestimated diseases," Rheumatology, vol. 50, no. 11, pp. 2029-2034, 2011.

[3] S. C. Shiboski, C. H. Shiboski, L. A. Criswell et al., "American College of rheumatology classification criteria for Sjögren's syndrome: a data-driven, expert consensus approach in the 
Sjögren's International Collaborative Clinical Alliance cohort," Arthritis Care and Research, vol. 64, no. 4, pp. 475-487, 2012.

[4] C. Vitali, S. Bombardieri, R. Jonsson et al., "Classification criteria for Sjögren's syndrome: a revised version of the European criteria proposed by the American-European Consensus Group," Annals of the Rheumatic Diseases, vol. 61, no. 6, pp. 554$558,2002$.

[5] C. H. Shiboski, S. C. Shiboski, R. Seror et al., "2016 American College of Rheumatology/European League Against Rheumatism classification criteria for primary Sjogren's syndrome A consensus and data-driven methodology involving three international patient cohorts," Annals of the Rheumatic Diseases, vol. 76, no. 1, pp. 9-16, 2016.

[6] S. Costa, I. Quintin-Roué, A. Lesourd et al., "Reliability of histopathological salivary gland biopsy assessment in Sjögren's syndrome: a multicentre cohort study," Rheumatology, vol. 54, no. 6, pp. 1056-1064, 2015.

[7] Y. Takagi, Y. Kimura, H. Nakamura, M. Sasaki, K. Eguchi, and T. Nakamura, "Salivary gland ultrasonography: can it be an alternative to sialography as an imaging modality for Sjögren's syndrome?" Annals of the Rheumatic Diseases, vol. 69, no. 7, pp. 1321-1324, 2010.

[8] R. K. Niemelä, R. Takalo, E. Pääkkö et al., "Ultrasonography of salivary glands in primary Sjögren's syndrome. A comparison with magnetic resonance imaging and magnetic resonance sialography of parotid glands," Rheumatology, vol. 43, no. 7, pp. 875-879, 2004.

[9] G. G. Song and Y. H. Lee, "Diagnostic accuracies of sialography and salivary ultrasonography in Sjögren's syndrome patients: a meta-analysis," Clinical and Experimental Rheumatology, vol. 32, no. 4, pp. 516-522, 2014.

[10] N. Luciano, C. Baldini, G. Tarantini et al., "Ultrasonography of major salivary glands: a highly specific tool for distinguishing primary Sjögren's syndrome from undifferentiated connective tissue diseases," Rheumatology (United Kingdom), vol. 54, no. 12, pp. 2198-2204, 2016.

[11] C. Baldini, N. Luciano, G. Tarantini et al., "Salivary gland ultrasonography: a highly specific tool for the early diagnosis of primary Sjögren's syndrome," Arthritis Research and Therapy, vol. 17, no. 1, article no. 146, 2015.

[12] V. Milic, R. Petrovic, I. Boricic et al., "Ultrasonography of major salivary glands could be an alternative tool to sialoscintigraphy in the American-European classification criteria for primary Sjögren's syndrome," Rheumatology, vol. 51, no. 6, pp. 1081-1085, 2012.

[13] S. Das, D. Huynh, H. Yang, A. Ceponis, and A. Kavanaugh, "Salivary gland ultrasonography as a diagnostic tool for secondary Sjögren syndrome in rheumatoid arthritis," Journal of Rheumatology, vol. 42, no. 7, pp. 1119-1122, 2015.

[14] D. S. Hammenfors, J. G. Brun, R. Jonsson, and M. V. Jonsson, "Diagnostic utility of major salivary gland ultrasonography in primary Sjögren's syndrome," Clinical and Experimental Rheumatology, vol. 33, no. 1, pp. 56-62, 2015.

[15] X. Zhang, S. Zhang, J. He et al., "Ultrasonographic evaluation of major salivary glands in primary Sjögren's syndrome: comparison of two scoring systems," Rheumatology, vol. 54, no. 9, pp. 1680-1687, 2015.

[16] E. Theander and T. Mandl, "Primary Sjögren’s syndrome: diagnostic and prognostic value of salivary gland ultrasonography using a simplified scoring system," Arthritis Care and Research, vol. 66, no. 7, pp. 1102-1107, 2014.
[17] D. Cornec, S. Jousse-Joulin, A. Saraux, and V. DevauchellePensec, "Salivary gland ultrasound to diagnose Sjögren's syndrome: a claim to standardize the procedure," Rheumatology, vol. 54, no. 1, pp. 199-200, 2015.

[18] J. Bamber, D. Cosgrove, C. F. Dietrich et al., "EFSUMB guidelines and recommendations on the clinical use of ultrasound elastographypart 1: basic principles and technology," Ultraschall in der Medizin, vol. 34, no. 2, pp. 169-184, 2013.

[19] D. Cosgrove, F. Piscaglia, J. Bamber et al., "EFSUMB guidelines and recommendations on the clinical use of ultrasound elastography.part 2: clinical applications," Ultraschall in der Medizin, vol. 34, no. 3, pp. 238-253, 2013.

[20] A. Knopf, B. Hofauer, K. Thürmel et al., "Diagnostic utility of acoustic radiation force impulse (ARFI) imaging in primary Sjoegren's syndrome," European Radiology, vol. 25, no. 10, pp. 3027-3034, 2015.

[21] A. Hočevar, A. Ambrožič, B. Rozman, T. Kveder, and M. Tomšič, "Ultrasonographic changes of major salivary glands in primary Sjögren's syndrome. Diagnostic value of a novel scoring system," Rheumatology, vol. 44, no. 6, pp. 768-772, 2005.

[22] A. F. Badea, A. T. Szora, E. Ciuleanu et al., "ARFI quantitative elastography of the submandibular glands. Normal measurements and the diagnosis value of the method in radiation submaxillitis," Medical Ultrasonography, vol. 15, no. 3, pp. 173179, 2013.

[23] D. Cornec, S. Jousse-Joulin, J.-O. Pers et al., "Contribution of salivary gland ultrasonography to the diagnosis of Sjögren's syndrome: toward new diagnostic criteria?" Arthritis and Rheumatism, vol. 65, no. 1, pp. 216-225, 2013. 


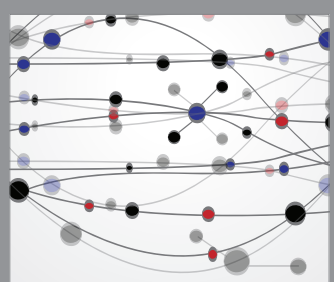

The Scientific World Journal
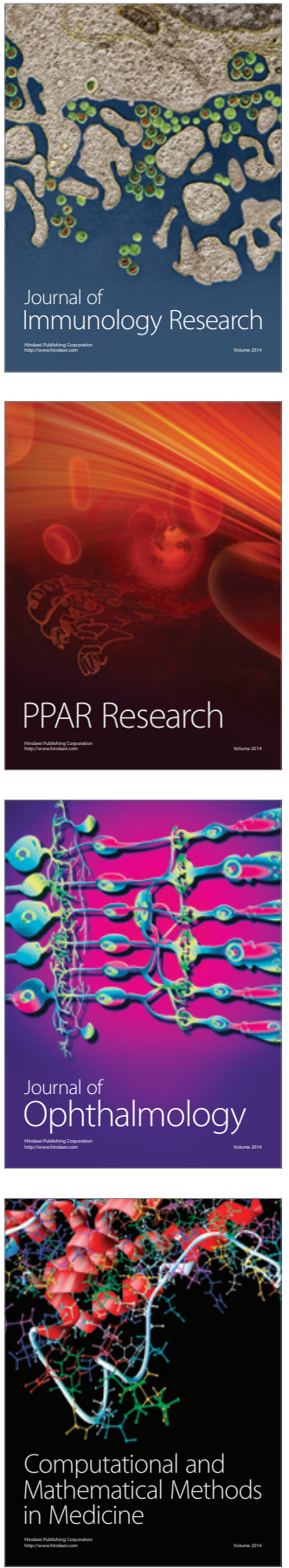

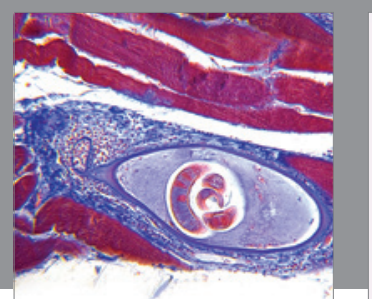

Gastroenterology Research and Practice

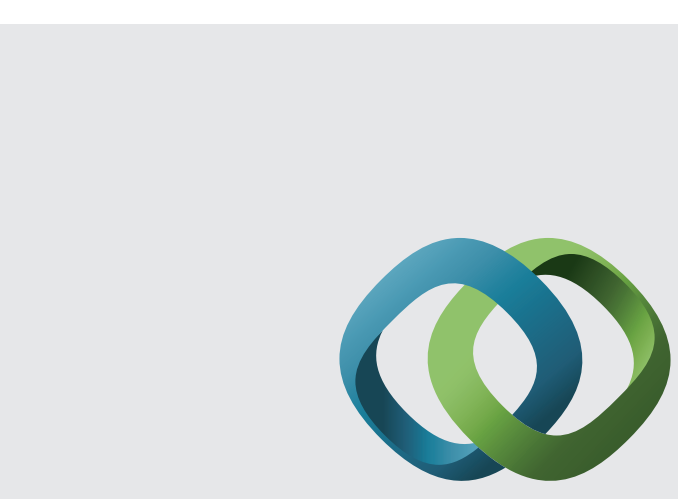

\section{Hindawi}

Submit your manuscripts at

http://www.hindawi.com
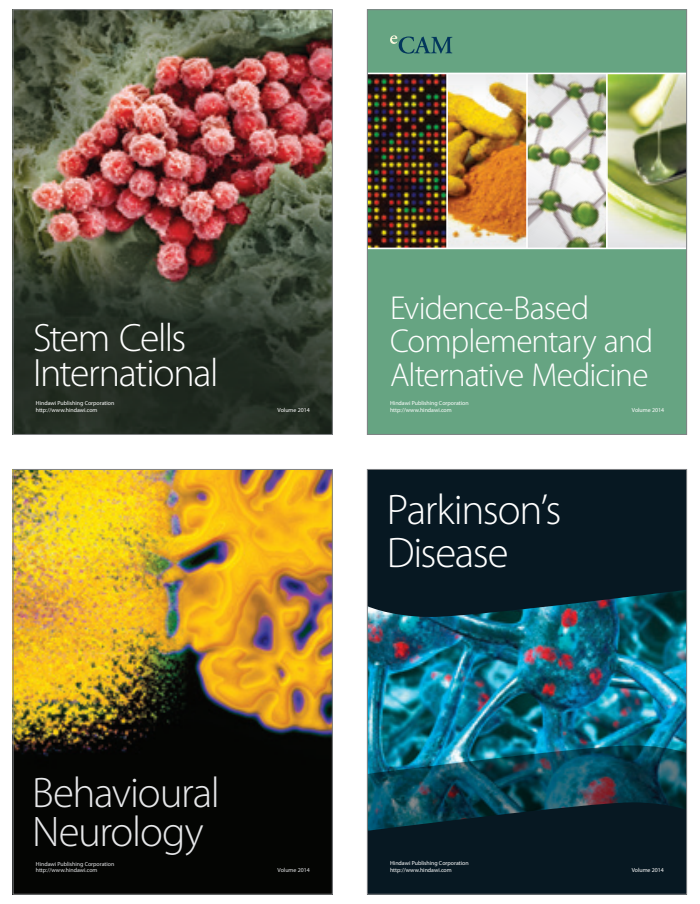
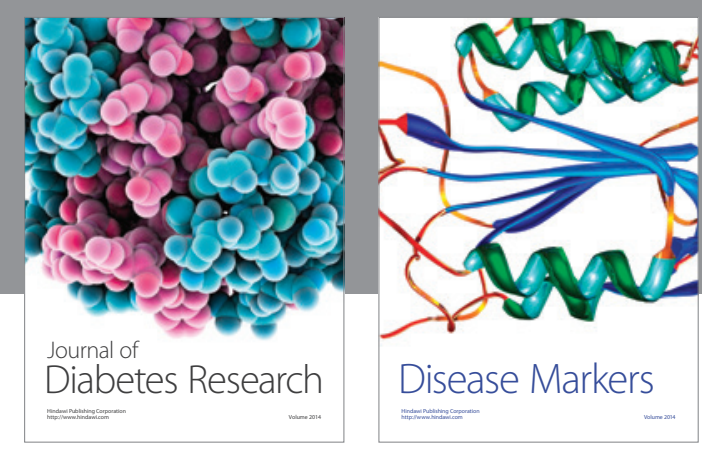

Disease Markers
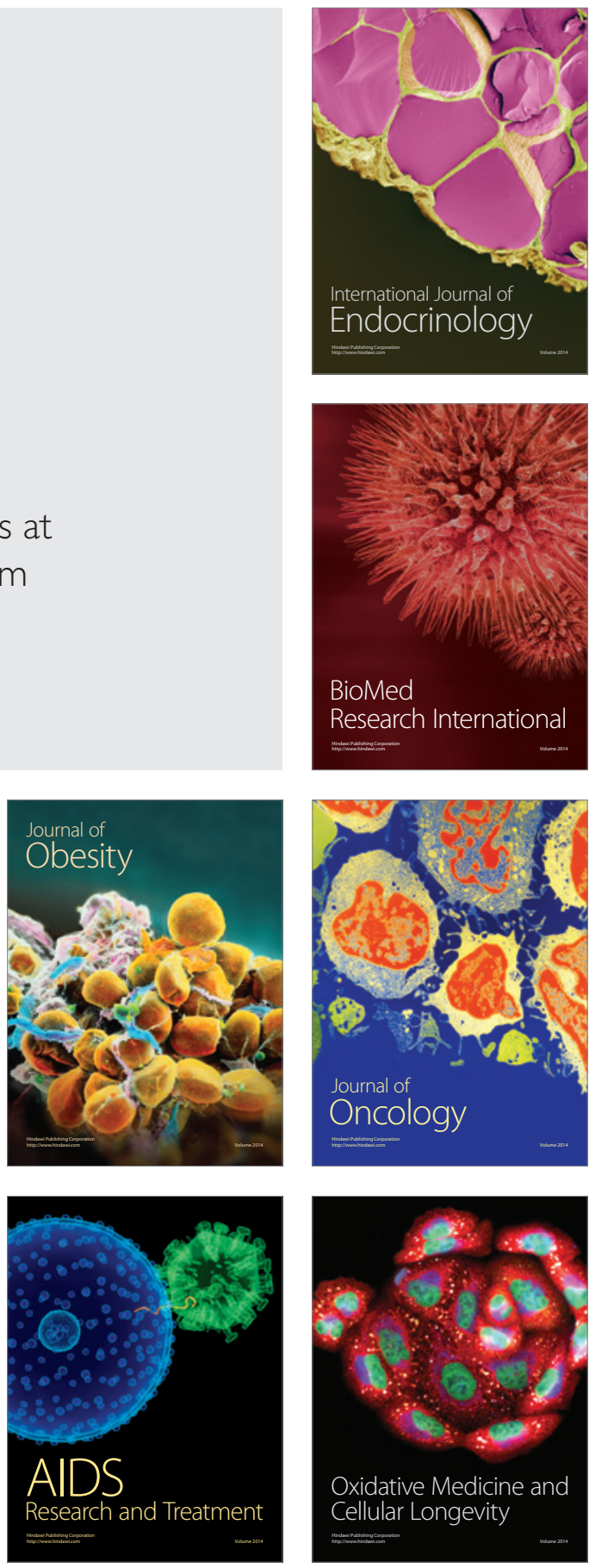